\title{
A Modified Total Crossing Time Model of Bidirectional Pedestrians at Signalized Crosswalks
}

\author{
Zhanzhong Wang, ${ }^{1}$ Liying Zhao, ${ }^{1}$ Ningbo Cao, ${ }^{1}$ Yue Lu, ${ }^{1}$ and Mingtao Chen ${ }^{2}$ \\ ${ }^{1}$ College of Transportation, Jilin University, Renmin Street 5988, Changchun, Jilin, China \\ ${ }^{2}$ Hualan Design \& Consulting Group, No. 39, Huadong Road, Nanning, Guangxi 530000, China \\ Correspondence should be addressed to Ningbo Cao; 819868226@qq.com
}

Received 18 November 2016; Revised 11 February 2017; Accepted 20 February 2017; Published 22 March 2017

Academic Editor: Stefan Balint

Copyright (c) 2017 Zhanzhong Wang et al. This is an open access article distributed under the Creative Commons Attribution License, which permits unrestricted use, distribution, and reproduction in any medium, provided the original work is properly cited.

\begin{abstract}
Since crosswalk width and pedestrian green time directly affect the safety of signalized crosswalks, modeling an exact total crossing time model to estimate those two variables is imperative. The total crossing time needed by a group of pedestrians to cross a signalized crosswalk contains the discharge time and the crossing time. The discharge time depends primarily on the maximum queue length, which is determined by pedestrian arrival rate, red interval, waiting position distribution, and the crosswalk width. Crossing time increases when interactions between bidirectional pedestrian flows become more serious. Thus, quantifying the impacts of the start-up process on the discharge time and the effects of the interactions on the crossing time is a prerequisite for optimizing the design of signalized crosswalks. This paper establishes a modified total crossing time model consisting of modified pedestrian discharge and crossing time. Discharge time is modeled by applying traffic wave theory, and crossing time is modeled based on drag force theory. The proposed models provide guidance for the design of crosswalk width and pedestrian green intervals.
\end{abstract}

\section{Introduction}

An accurate total crossing time model of bidirectional pedestrian flow plays a significant role in improving pedestrian safety and crosswalk design. So, modeling the relationship between total crossing time, number of pedestrians, and crosswalk width becomes a research focus. Over the past decades, many kinds of methods have been adopted to model the total crossing time at signalized crosswalks. However, few studies have focused on the interaction process of bidirectional pedestrians. Most of the existing studies tried to explore the effect of bidirectional pedestrians on the crossing time based on several assumptions, such as the motion of pedestrians exhibiting uniform deceleration when they meet pedestrians moving in the opposite direction. However, the interaction between bidirectional pedestrians is more complex than that.

As described in the Pedestrian chapter of the Highway Capacity Manual [1], the total crossing time can be estimated by (1). In (1), 3.2 represents the initial start-up lost time, and it is contained within the discharge time in this paper. The total crossing time is a function of the bidirectional pedestrian flow, the bidirectional effect, and the crosswalk width:

$$
\begin{aligned}
& 3.2+\frac{L}{S_{p}}+2.7 * \frac{N_{\text {ped }}}{w}, \quad \text { for } w>10 \mathrm{ft} \\
& 3.2+\frac{L}{S_{p}}+0.27 * N_{\text {ped }}, \quad \text { for } w \leq 10 \mathrm{ft} .
\end{aligned}
$$

Based on the method of the HCM (1), Golani and Damti put forward a modified model to estimate total crossing time [2]. They took the effect of bidirectional pedestrian flow on the total crossing time into consideration and developed a model including start-up lost time, average walking speed, and pedestrian headway. However, it was difficult to model the interaction in the proposed model. Lam et al. [3] concluded that bidirectional flow ratios have remarkable effects on the average walking speed and the maximum pedestrian capacity. However, their study ignored the impacts of different walkway widths on the average walking speed and the maximum pedestrian flow. Alhajyaseen et al. [4] 


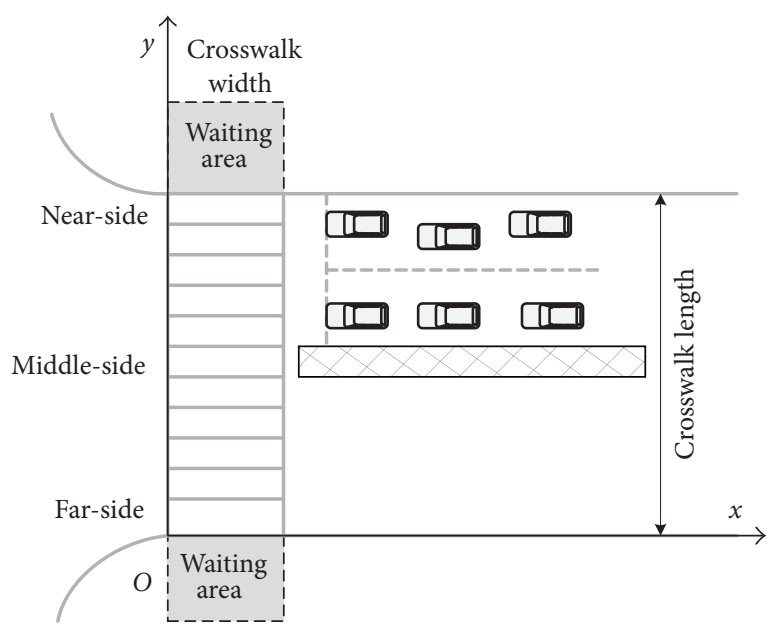

(a) Definition of crosswalk geometry

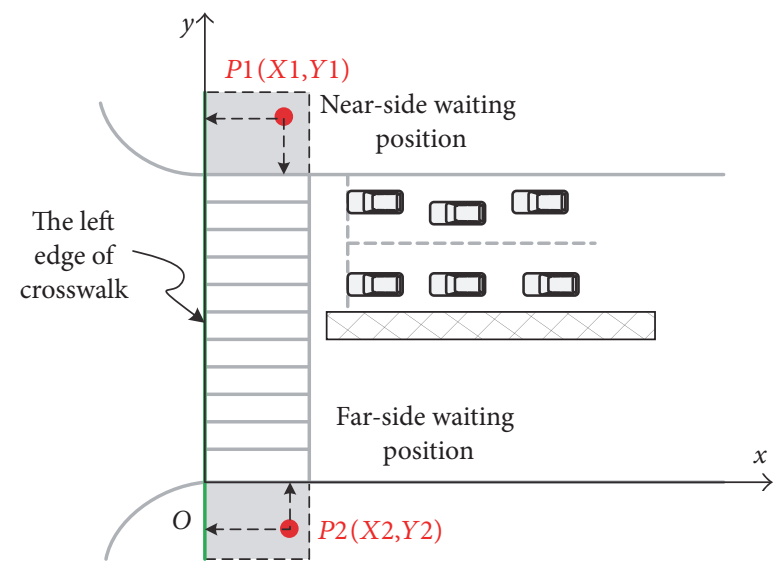

(b) Definition of waiting positions

FIgURE 1: Definition of parameters.

investigated the effects of bidirectional flow ratios on the maximum pedestrian flow on crosswalks. Marisamynathan and Vedagiri [5] divided the total crossing delay into a waiting delay and a crossing delay, according to the crossing process, and finally modeled the total crossing delay and the total crossing time. Zhang et al. [6] evaluated pedestrian crossing time by considering the effects of crosswalk width and signal timing on crossing speed. Xu and Wang [7] used the conflict theory and the critical-gap accepted theory to model pedestrian crossing time when considering the effects of left-turning cars on pedestrian crossing. Zhao and Guo [8] built up an estimating model of crossing times. Their model is divided into a time model for the pedestrian crossing waiting area based on wave theory and a time model for the pedestrian crossing at crosswalks. A pedestrian total crossing time, equal to the sum of discharge time and crossing time, was established by Alhajyaseen et al. [9-11]. They proposed a macroscopic method. For modeling discharge time, the traffic wave theory was adopted. Then, they applied drag force theory to model crossing time. However, their models contain two shortcomings. Firstly, pedestrians were assumed to be uniformly distributed in the waiting area. Secondly, the interaction between bidirectional pedestrians was assumed to be fixed. Both assumptions cannot conform to reality. There is no doubt that these models are useful but they still contain some disadvantages when referring to bidirectional pedestrians.

This paper represents an improvement over the previous methods of Alhajyaseen et al. [9-11] and aims to improve the total crossing time model from two aspects. The purpose of this study is to establish modified discharge time model and modified crossing time. Firstly, the pedestrian waiting position distribution model, taking the crosswalk geometry and pedestrian density into consideration, is established based on actual survey data from seven survey sites. Secondly, the maximum queue length of pedestrians is calculated using the traffic wave model, and then the discharge time model of pedestrians is proposed. Thirdly, considering the nature of bidirectional pedestrian flow, a modified crossing time model based on the drag force theory is formulated as a function of the number of pedestrians, the directional ratio, and the crosswalk width. Finally, the parameters of the models are calibrated, the sensitivity of the proposed models to bidirectional pedestrian factors and crosswalk width are analyzed, and the established models are validated in a comparison with the HCM and Alhajyaseen models.

\section{Data Collection and Methods}

2.1. Parameters Definition. Pedestrians modify their behavior according to the traffic environment and interactions with other pedestrians. In order to ensure their safety, pedestrians tend to cross in the middle of crosswalks, and the edges of crosswalks are used less frequently. According to data collected on pedestrians' waiting positions in the crosswalk waiting area during the red light period, the frequencies of pedestrians in different positions were analyzed. It is abundantly clear that the crosswalk geometry and volume of pedestrians have a significant influence on the waiting positions. The geometric characteristics of the crosswalk, pedestrian density, and waiting positions are defined in Figure 1.

2.1.1. Geometric Characteristics of Crosswalk. As shown in Figure 1(a), the geometric characteristics include crosswalk width and crosswalk length.

2.1.2. Waiting Positon and Average Waiting Position. The coordinate system is established in Figure 1(a), where $O$ is the origin. As shown in Figure 1(b), the coordinates of pedestrians in the waiting area are defined as the waiting position (e.g., $P 1$ and $P 2$ ). The average waiting position represents the average $x$-coordinate across all the observed waiting pedestrians at a single survey site.

2.1.3. Waiting Pedestrian Probability. $F_{i}(i=1,2,3,4, \ldots)$ is used to represent the probability that a pedestrian will stand within a particular interval in the waiting area. $N$ represents 
TABLE 1: Geometric characteristics of study sites.

\begin{tabular}{|c|c|c|c|c|c|c|c|}
\hline \multirow{2}{*}{ Crosswalk analyzed } & \multicolumn{2}{|c|}{ Gongnong Road } & \multicolumn{2}{|c|}{ Ziyou Road } & \multicolumn{2}{|c|}{ Chongqing Road } & \multirow{2}{*}{$\begin{array}{c}\text { Renmin Street } \\
\text { West }\end{array}$} \\
\hline & East & North & East & North & East & South & \\
\hline Observation time (p.m. two days) & $13: 00-15: 30$ & 13:00-15:30 & $13: 00-15: 30$ & $13: 00-15: 30$ & $13: 00-15: 30$ & 13:00-15:30 & $13: 00-15: 30$ \\
\hline Crosswalk width/m & 8 & 7 & 7 & 8 & 10 & 4 & 6 \\
\hline Crosswalk length/m & 43 & 29 & 23 & 40 & 44 & 15 & 18 \\
\hline Sample size of pedestrians & 734 & 572 & 542 & 468 & 670 & 800 & 624 \\
\hline Total & & & & 4410 & & & \\
\hline
\end{tabular}

the total number of observed waiting pedestrians at one survey site. $N_{i}(i=1,2,3,4, \ldots)$ represents the number of waiting pedestrians. For example, $i=1$ means that the $x$ coordinate is located in the $[0,1]$ range and $i=2$ means that the $x$-coordinate is located in the $[1,2]$ range. $F_{i}$ equals the ratio of $N_{i}$ to $N$.

2.1.4. Waiting Pedestrian Density. When pedestrians enter the waiting area, they choose the appropriate waiting position according to the waiting pedestrian density. This equals the ratio of waiting pedestrians $N_{w}$ arriving at the waiting area during one green interval to the waiting area.

2.2. Study Sites. In order to study the influences of different factors on pedestrians' waiting positions in the waiting area, seven crosswalks at four signalized intersections were chosen as the study sites. All are located in Chang Chun in China, and all are in commercial centers, ensuring sufficient pedestrian samples were obtained to enable a thorough analysis of pedestrians' waiting behavior.

The geometric characteristics and sample sizes of pedestrians at the seven surveyed crosswalks are shown in Table 1. Series of traffic data were collected using video-processing technology at the seven crosswalks, which had different geometric characteristics and pedestrian volumes. Software based on a video image processing system was applied to extract pedestrian coordinates at every moment, and on the basis of the pedestrian coordinates, the distributions of pedestrian waiting positions in the waiting area were analyzed. Besides this, acceleration, velocity, discharge time $T_{d}$, crossing time $T_{c}$, and direction split ratio $r$ could all be recorded. $T_{d}$ represents the discharge time required for a pedestrian group to walk from the waiting area and step onto the crosswalk; $T_{c}$ represents the time needed to cross the crosswalk. All these data will be used to estimate parameters such as the drag coefficient $\left(C_{\text {adj }}\right)$.

\section{Development of Modified Discharge Time Model}

This paper is a modified model of Alhajyaseen models [10], so the development process of method established by Alhajyaseen is briefly introduced. The total crossing time was divided into two parts: discharge time $T_{d}$ and crossing time $T_{c}$. As shown in (2),

$$
T=T_{d}+T_{c},
$$

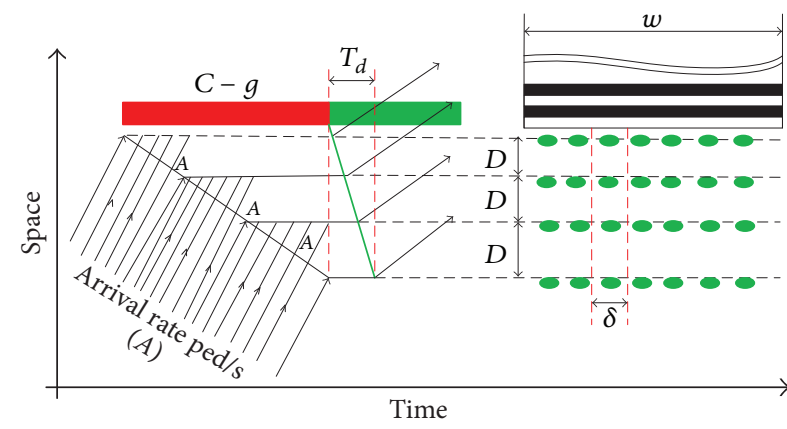

FIgURE 2: Pedestrian waiting and discharge mechanism.

where $T$ represents the total crossing time, $T_{d}$ is the discharge time needed for a pedestrian group to walk from the waiting area and step onto the crosswalk, and $T_{c}$ is the time needed for them to cross the crosswalk.

Discharge time $T_{d}$ represents the time from the first waiting pedestrian stepping into the crosswalk to the last waiting pedestrian stepping into the crosswalk. Crossing time $T_{c}$ is the necessary time to cross the crosswalk, because the discharge time $T_{d}$ is similar to the discharge time of cars, which is frequently modeled by shockwave theory. So, the shockwave theory also be used to model the discharge time. Start-up lost time $I$ is included in discharge time $T_{d}$ (Figure 2). By utilizing shockwave theory, the discharge time needed for the pedestrians in the longest queue to step into crosswalk can be expressed as

$$
\begin{aligned}
& T_{d} \\
& =\frac{\left((-\delta A / w) /\left(K_{j}-\delta A / w u_{s}\right)\right)(C-g)}{\left(Q_{d} /\left(Q_{d} / u_{0}-K_{j}\right)\right)-\left((-\delta A / w) /\left(K_{j}-\delta A / w u_{s}\right)\right)},
\end{aligned}
$$

where $A$ represents pedestrian arrival rate (ped/s); $\delta$ represents lateral distance $(\mathrm{m}) ; C$ represents cycle length $(\mathrm{s})$; $g$ represents pedestrian green interval (s); $Q_{d}$ represents pedestrian discharge rate (ped/s); $K_{j}$ represents jam density $\left(\mathrm{ped} / \mathrm{m}^{2}\right) ; u_{s}$ represents average speed of arriving pedestrians $(\mathrm{m} / \mathrm{s}) ; u_{0}$ represents free-flow speed $(\mathrm{m} / \mathrm{s}) ; w$ represents the crosswalk width $(\mathrm{m})$.

Crossing time $T_{c}$ is determined by pedestrian speed, which is influenced by the number of opposite pedestrians and crosswalk width. Because the interaction between bidirectional pedestrians is analogous to the fluid, the drag 
force theory is adopted to model the crossing time $T_{c}$. $T_{c}$ is expressed as

$$
T_{c}=\frac{\left(L_{c}-L_{0} / 2\right)}{\sqrt{u_{0}^{2}-C_{D_{\text {adj }}} P_{2} u_{0}^{2}\left(L_{c}-L_{0} / 2\right) / w}}+\frac{L_{0}}{2 u_{0}},
$$

where $L_{c}$ represents average trajectory length of the subject pedestrian demand $(\mathrm{m}) ; L_{0}$ is crosswalk length $(\mathrm{m}) ; C_{D_{\text {adi }}}$ is adjusted drag coefficient; $u_{0}$ represents average speed of discharge pedestrians $(\mathrm{m} / \mathrm{s}) ; w$ represents the crosswalk width (m); $P_{2}$ represents the number of subject pedestrians.

However, Alhajyaseen models had several shortcomings: (1) Pedestrian arrival rate is assumed to be uniform. Moreover, pedestrians are assumed to arrive in a unit of "pedestrian row" per second. (2) The distribution of pedestrian waiting positions is assumed to be uniform. (3) All pedestrians occupy the same fixed space. (4) The deceleration of the subject pedestrian flow is assumed to be invariable. The development of their model is based on this assumption, so Newton's Second Law is selected to model, which ignored the complex interactions between bidirectional pedestrians.

Frequently, Alhajyaseen models are proposed to define the required crosswalk width under different pedestrian demand volumes and estimate pedestrian crossing and discharge times to pedestrian signal timing optimization. However, these above shortcomings result into unreasonable computation, such as too long signal timing or too narrow pedestrian crosswalk.

Besides, this is because pedestrians prefer to obey the traffic rules and many crosswalks with directions are set to separate bidirectional pedestrians to reduce the interactions between bidirectional pedestrians' flow in Japan. In China, pedestrians prefer to wait in the middle region of waiting area; that is, the edges of the waiting area and crosswalk have lower utilization. Besides, there are larger pedestrian volume and complex interactions between bidirectional pedestrians' flows, and the design manual is very rough in China. All of these differences result in unreasonable crosswalk design and signal timing in China. So it is necessary to make some improvement of the method to overcome these shortcomings.

\subsection{Factors Affecting Distribution of Pedestrian Waiting Posi-} tions. According to the collected pedestrian waiting positions, the pedestrian waiting frequencies in different waiting positions were modeled. When a pedestrian arrives in the waiting area, he/she will choose a waiting position according to the pedestrian density in the waiting area. To model the distribution of pedestrian waiting positions, firstly, the effects of crosswalk geometry and waiting pedestrian density are analyzed.

As shown in Figure 3(a), the relationship between crosswalk width and average waiting position is approximately linear. High $R^{2}$ values indicate a significant relationship between crosswalk width and average waiting position. The average waiting position in every waiting area increases with the crosswalk width. Similarly, Figure 3(b) shows that there is a significant relationship between crosswalk length and average waiting position. $R^{2}$ is 0.89 , which indicates that the average waiting position in the waiting area increases with the crosswalk length.

In the regression analysis, the $R^{2}$ value is larger than 0.9 , which indicates that the curve fit is good. As shown in Figure 3(c), the relationship between pedestrian density and average waiting position conforms to a linear relationship in one waiting area, which means that the average waiting position increases with pedestrian density. The same method is applied to analyze the relationship between pedestrian density and average waiting position at the other study sites. The minimum adjusted $R^{2}$ value at the other study sites is 0.88 , which suggests that the average waiting position indeed increases with the waiting pedestrian density.

In conclusion, the crosswalk geometry and waiting pedestrian density can be selected as variables for modeling the distribution of waiting positions.

3.2. Development and Validation of Waiting Position Distribution Model. Figure 4 shows the raw distributions of pedestrian waiting positions at each waiting area of the seven observed crosswalks. As shown in Figure 4, the peaks of the histograms in the waiting areas shift to the right, which means that the waiting positions increase. To capture the different characteristics of the shapes of these waiting position distributions, an appropriate and flexible model is needed.

Walck [12] indicated that the Weibull distribution could be used to represent various random distributions through the adjustment of the shape and scale parameters. Therefore, the Weibull distribution is selected to model the waiting position distributions in the waiting area. Based on the established waiting position distributions, the discharge time model is obtained. The density probability function of the Weibull distribution is shown in

$$
f(x, \alpha, \beta)=\frac{\alpha}{\beta}\left(\frac{x}{\beta}\right)^{\alpha-1} e^{-(x / \beta)^{\alpha}},
$$

where $f$ is the probability function of the Weibull distribution, $\alpha$ is the shape parameter which controls the shape of the distribution, and $\beta$ is the scale parameter which controls the mean and standard deviation of the distribution.

The distributions of pedestrian waiting positions obey the Weibull distribution. According to the above analyses, the crosswalk geometry and waiting pedestrians density are the key factors influencing the waiting position distribution. Thus, they are selected as the variables for modeling the distribution, with the shape parameter $\alpha$ and scale parameter $\beta$ of the Weibull distribution modeled as functions of these three variables, as shown in

$$
\begin{aligned}
& \alpha=f(w, l, k)=\lambda_{1,0}+\lambda_{1,1} w+\lambda_{1,2} l+\lambda_{1,3} k \\
& \beta=f(w, l, k)=\lambda_{2,0}+\lambda_{2,1} w+\lambda_{2,2} l+\lambda_{2,3} k,
\end{aligned}
$$

where $\lambda_{1,0}, \lambda_{1,1}, \ldots, \lambda_{1, n}$ and $\lambda_{2,0}, \lambda_{2,1}, \ldots, \lambda_{2, n}$ are the model coefficients, $w$ is the crosswalk width (m), $l$ is the crosswalk length $(\mathrm{m})$, and $k$ is the density of waiting pedestrians $\left(\mathrm{ped} / \mathrm{m}^{2}\right)$.

The coefficients of the shape parameter $\alpha$ and scale parameter $\beta$ are estimated by the maximum likelihood 


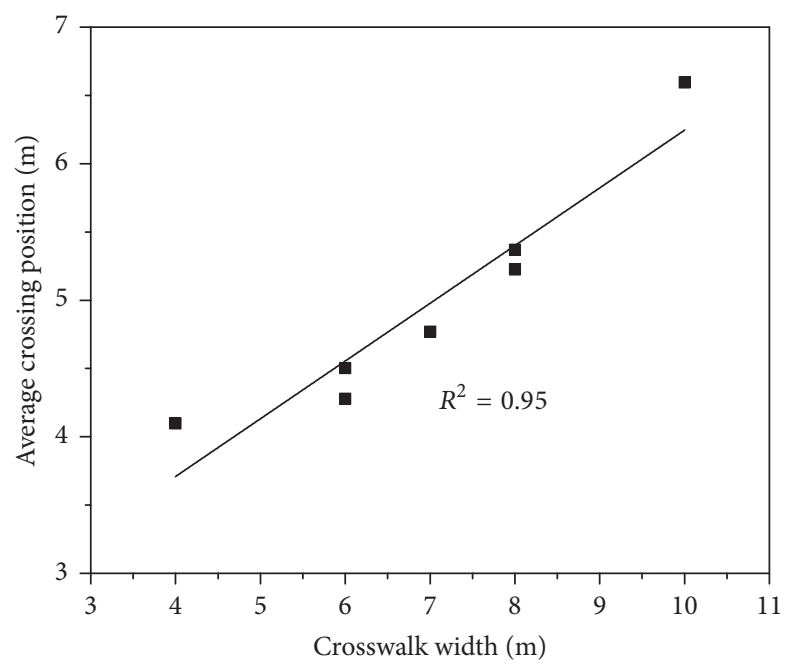

(a)

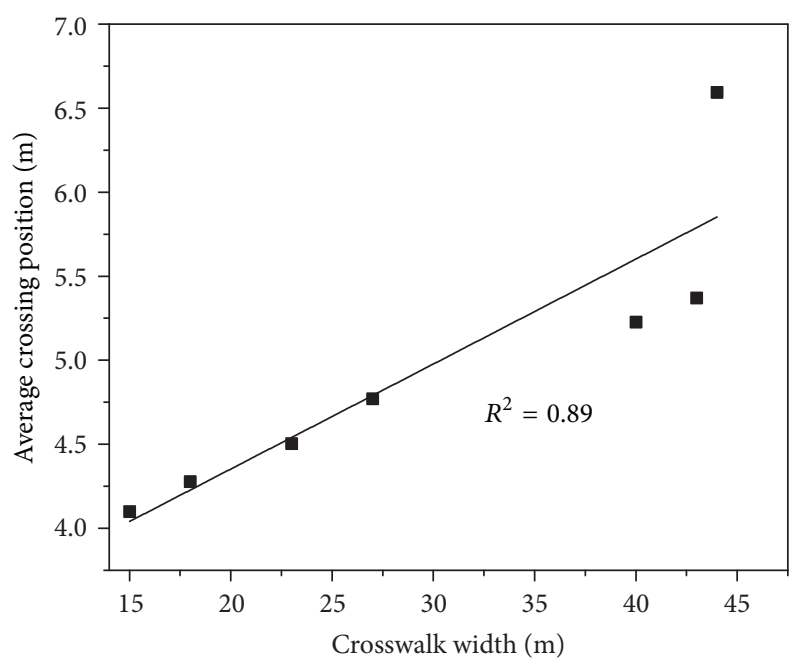

(b)

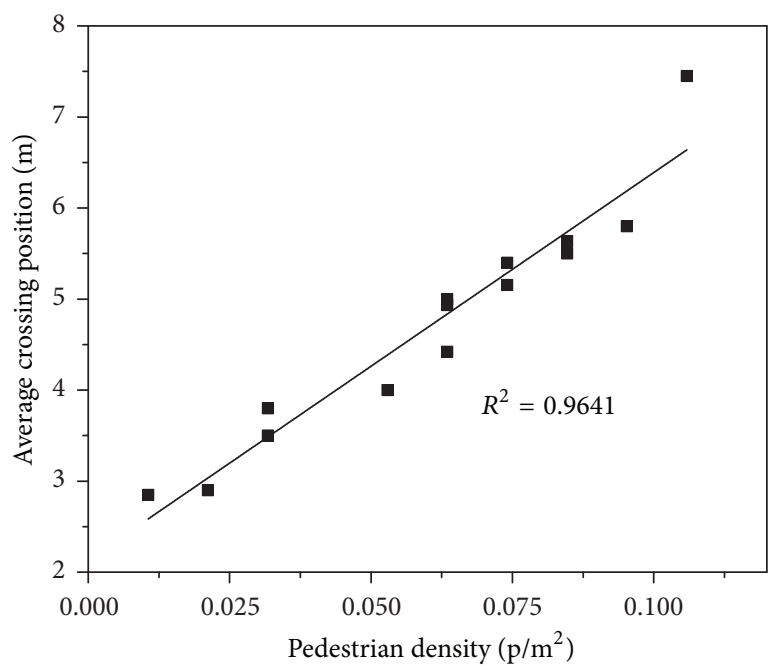

(c)

FIGURE 3: Relationship between factors and average waiting position.

method. The resulting functions are shown in (7). The adjusted $R^{2}$ of $\alpha$ and $\beta$ are 0.76 and 0.71 , respectively:

$$
\begin{aligned}
& \alpha=6.89-0.43 w+0.044 l-1.72 k \\
& \beta=2.31-0.49 w+0.089 l-11.6 k .
\end{aligned}
$$

Model validation is next conducted to confirm whether the established model adequately represents the distribution of waiting positions in the waiting area. In comparing the observed distributions of pedestrian waiting positions and the estimated results, Hongqi Street's east crosswalk is selected as the validation site, because of its longer crosswalk length, width, and pedestrian volumes. The crosswalk geometry was shown in Table 1. As shown in Figure 5, based on two sample $t$-tests for the difference in waiting positions, at a $95 \%$ confidence level there is no significant difference.

3.3. Modeling Discharge Time. The discharge time $T_{d}$ relies primarily on the maximum queue length, and then the maximum queue length is determined by pedestrian arrival rate, red interval, distribution of waiting positions, and crosswalk width. The longest queue is located in the position with the biggest waiting probability, as shown in Figure 6. The discharge time is the time needed for all the waiting pedestrians to step into the crosswalk. As analyzed in the previous section, the distribution of waiting positions of the pedestrians arriving in one-minute interval obeys the Weibull distribution. When all the pedestrians in the longest queue have stepped into the crosswalk, all the waiting pedestrians in the entire waiting area will have discharged entirely. In other words, the discharge time of the pedestrians in the longest queue represents the discharge time of all the waiting pedestrians. Thus, how to calculate the discharge time of the pedestrians in the longest queue according to the waiting positions distribution model is the key step.

Traffic wave theory is adopted for modeling the discharge time. Start-up lost time is included in the discharge time $T_{d}$. $A$ represents the pedestrian arrival rate and is assumed to be 


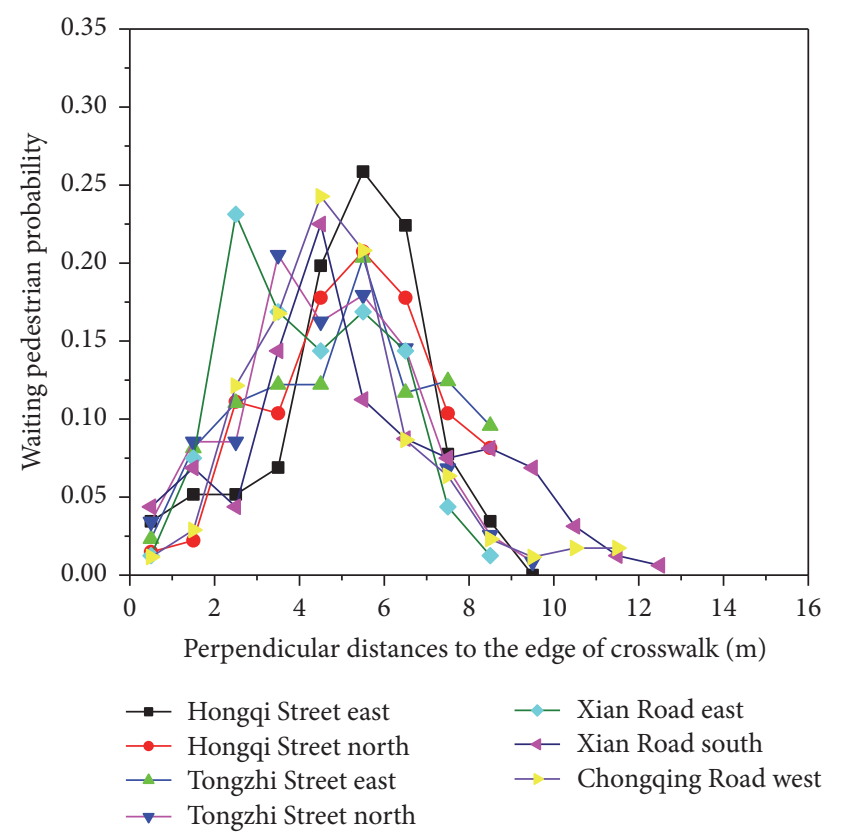

FIgURE 4: Pedestrian waiting position distributions.

uniform. Moreover, pedestrians are assumed to arrive in units of "pedestrian rows" per second. Thus, the pedestrian arrival rate at the position with the biggest waiting probability is $A *$ $P_{\max }$, where $P_{\max }$ is the biggest waiting probability and can be calculated using (5). Using traffic wave theory, the discharge time needed for the pedestrians in the longest queue to step into the crosswalk can be expressed as (9), whose derivation is shown in (8):

$$
\begin{aligned}
& \left(\frac{Q_{d}}{Q_{d} / v_{0}-K_{j}}\right) T_{d}=\left(\frac{-A P_{\max }}{K_{j}-A P_{\max } / u_{s}}\right)(C-g) \\
& T_{d}=\frac{\left(-A P_{\max } /\left(K_{j}-A P_{\max } / u_{s}\right)\right)(C-g)}{\left(Q_{d} /\left(Q_{d} / v_{0}-K_{j}\right)\right)},
\end{aligned}
$$

where $A$ represents the pedestrian arrival rate (ped/s), $P_{\max }$ is the biggest waiting probability in the waiting area, $C$ is the cycle length (s), $g$ is the pedestrian green interval (s), $Q_{d}$ is the pedestrian discharge rate (ped/s), $K_{j}$ is the jam density ( $\left.\mathrm{ped} / \mathrm{m}^{2}\right), u_{s}$ is the average speed of arriving pedestrians $(\mathrm{m} / \mathrm{s})$, and $v_{0}$ is the average speed of discharge of pedestrians $(\mathrm{m} / \mathrm{s})$.

\section{Development of Crossing Time Model}

4.1. Analysis of Speed and Acceleration. To analyze the interaction between bidirectional pedestrians, their speed and acceleration first need to be analyzed. Note that the average speeds and acceleration of pedestrians moving in one direction are recorded at every moment in every green interval. Because we need to analyze the interaction between pedestrians moving in opposing directions, their volume needs to be large enough to form "lanes." At every study site, the same analytical method was used. However, since

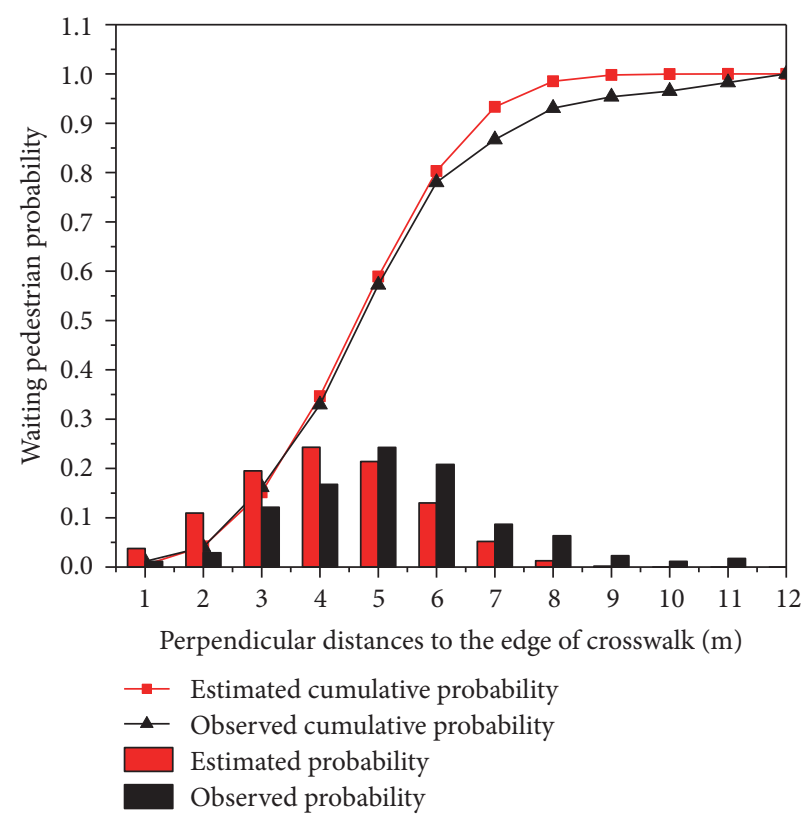

FIGURE 5: Observed and estimated pedestrian waiting positions.

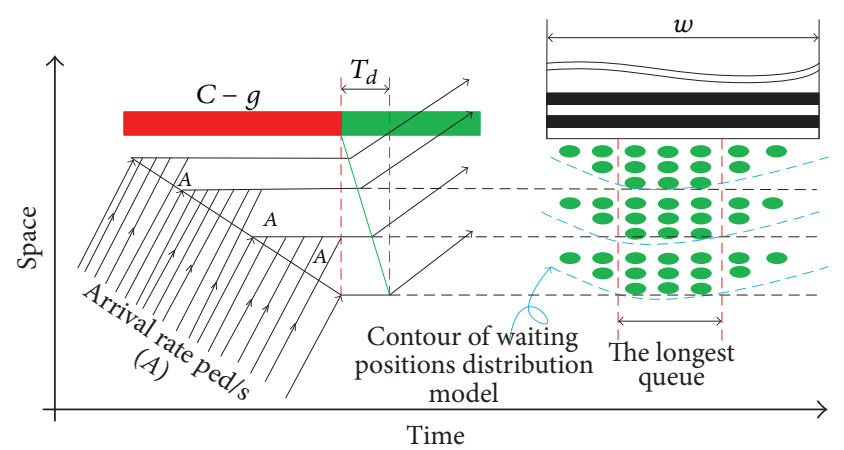

FIgURE 6: Pedestrian waiting and clearance mechanism.

presenting all the analysis results in this paper would be difficult, we choose just a representative example to explain the interaction between bidirectional pedestrians. Figure 7 shows the variations in the subject pedestrians' speeds and acceleration in one green interval at Ziyou Road's north crosswalk. As shown in Figure 7(a), the subject pedestrians maintained the same speed before they met pedestrians moving in the opposite direction (opposing pedestrians). Then, they began to slow down to a minimum speed. After the first subject pedestrian crossed the interaction zone, all subject pedestrians began to accelerate until the last one crossed the interaction zone. The change in acceleration is a reflection of the level of interaction. Figure 7(b) shows that the deceleration of the subject pedestrians increases gradually as the interaction zone expands. That is, the interaction between bidirectional pedestrians becomes more and more significant. After the first of the focal pedestrians crosses the interaction zone, they all begin to accelerate, and the acceleration increases gradually with time, indicating that the interaction between the bidirectional pedestrians reduces. 
TABLE 2: Relation between shape and drag force.

\begin{tabular}{|c|c|c|c|c|c|}
\hline Shape and flow & Form drag & Skin friction & Shape and flow & Form drag & Skin friction \\
\hline ב三 & $0 \%$ & $100 \%$ & & $\sim 90 \%$ & $\sim 10 \%$ \\
\hline$=$ & $\sim 10 \%$ & $\sim 90 \%$ & & $100 \%$ & $0 \%$ \\
\hline
\end{tabular}

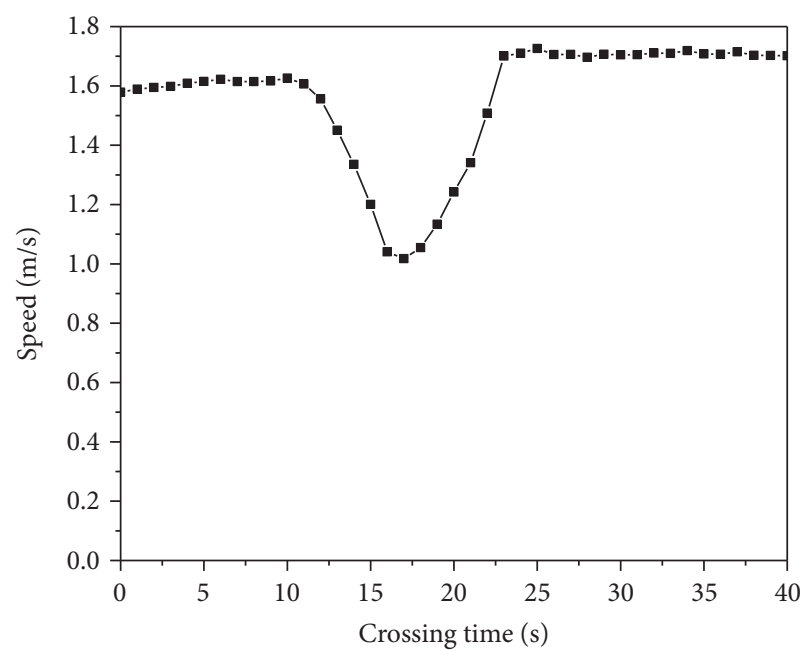

(a)

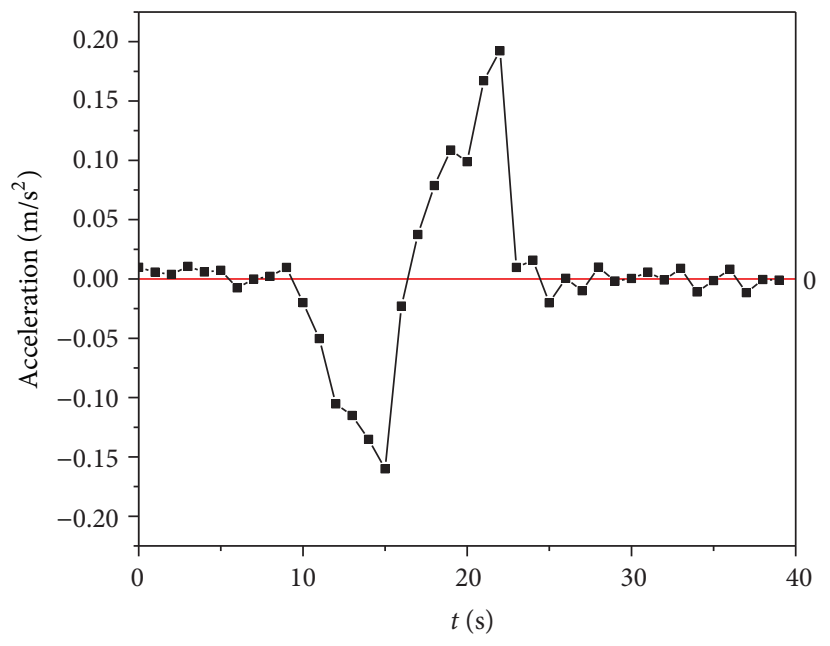

(b)

FIgURE 7: Variation in focal pedestrians' speed and acceleration.

4.2. Modeling Crossing Time. The drag force is a force that acts on an object and constrains its movement, causing resistance. When an object moves in a fluid, such as water, the resistance acting on the object is called hydrodynamic drag. When the focal and opposing pedestrians meet each other, resistance from the opposing pedestrian flow will act on the focal pedestrians. The interaction of bidirectional pedestrians is a complicated phenomenon and one that is hard to model and explain. Drag force theory is a well-developed method of modeling interaction. A drag force model was proposed by Pugh $[13,14]$ in which the drag force was expressed as in

$$
D=C_{d} q A_{p}
$$

where $D$ is the drag force $\left(\mathrm{kg} \mathrm{m} / \mathrm{s}^{2}\right), C_{d}$ the drag coefficient (a dimensionless number), $q$ dynamic pressure (force per unit area), and $A_{p}$ cross-sectional area $\left(\mathrm{m}^{2}\right)$.

The dynamic pressure $q$ can be expressed as in (11) to (14):

$$
q=\frac{1}{2} \rho u^{2}
$$

where $\rho$ is the density of the fluid $\left(\mathrm{kg} / \mathrm{m}^{3}\right)$ and $u$ the speed of the subject relative to the fluid $(\mathrm{m} / \mathrm{s})$.
By substituting (11) into (10), the final drag force formula is expressed as in

$$
D=\frac{1}{2} C_{d} \rho u^{2} A_{p}
$$

Drag force is related to the shape of the subject and includes form drag and skin friction. The relation between the shape of the subject and the drag force is shown in Table 2.

The subject pedestrians are assumed to be spherical by Alhajyaseen and Nakamura [10,11], and the drag force acting upon them is shown in Figure 9(a). This assumption has one shortcoming. The drag force will stay the same because it is determined by the cross-sectional area $A_{p}$. As introduced in those studies, $A_{p}$ is estimated by

$$
A_{p} \approx \beta n
$$

where $A_{p}$ is the cross-sectional area of the focal pedestrian flow $\left(\mathrm{m}^{2}\right), \beta$ represents the average width of one pedestrian, and $n$ is the number of pedestrians in the focal pedestrian flow.

As shown in Figure 8, lane formation is a frequently occurring phenomenon with multiple pedestrians. The subject pedestrians pass the opposing pedestrians in a queue. In 


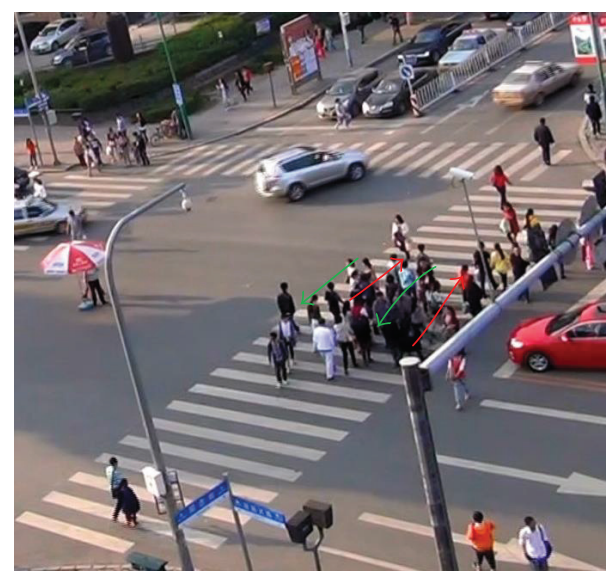

FIGURE 8: Lane formation in bidirectional pedestrian flow.

Alhajyaseen and Nakamura's model, pedestrians interact with each other one-to-one. In fact, when lane formation occurs in bidirectional pedestrian flow, the pedestrians in one "lane" walk like a team. Thus, the shape of the subject pedestrians looks more like cylindrical, and the drag force is as shown in Figure 9(b). With cylindrical shapes, the drag force is equal to the skin friction, and the skin friction increases progressively as the contact area increases. $A_{p}$ in (12) becomes the contact area. Figure 9(b) better represents this phenomenon (where the pedestrians in one "lane" walk like a team).

As analyzed in Section 4.1, as the interaction between the subject and opposing pedestrians becomes stronger, the interference received by the subject pedestrians will become more serious. In other words, the drag force from the opposing pedestrian flow will become larger over the interaction time. The deceleration of the focal pedestrian changes over time rather than staying the same (constant deceleration). Thus, the crossing time model needs to be modified so as to reconcile with the reality.

To apply the drag force theory to model the interactions between the focal and opposing pedestrian flows, three assumptions are made:

(i) Before the focal and opposing pedestrians meet each other, the speeds of both are assumed to be the free-flow speed, $v_{0}$, so that the speed of the focal pedestrians relative to the fluid becomes

$$
u=\left(v_{1}-\left(-v_{2}\right)\right)=v_{0}+v_{0}=2 v_{0} .
$$

(ii) $A_{p}$ in (12) represents the contact area and, because the contact area is proportional to the interaction length $l$, it is reasonable to replace $A_{p}$ with $l$.

(iii) The opposing pedestrian flow is regarded as a fluid and the focal pedestrian flow as a moving body. Thus, the density of the fluid can be expressed as in

$$
\rho=\frac{N_{2}}{w * l_{i}},
$$

where $w$ is the crosswalk width (m), $N_{2}$ is the number of opposing pedestrians, and $l_{i}$ is the maximum interaction length $(\mathrm{m})$, as shown in Figure 10(d).

After substituting (14) and (15) into (12), the drag force formula becomes

$$
D=\frac{1}{2} C_{d} \rho u^{2} A_{p}=\frac{1}{2} C_{d} \frac{N_{2}}{w * l_{i}} 4 v_{0}^{2} l=\frac{1}{2} C_{\text {adj }} \frac{N_{2}}{w * l_{i}} v_{0}^{2} l,
$$

where $C_{\text {adj }}$ is the adjusted drag coefficient, expressed as in

$$
C_{\text {adj }}=4 * C_{d} .
$$

Figure 10 illustrates the process for a platoon of pedestrians crossing a crosswalk. In Figure 10, the time from moment (a) to moment (b) equals the discharge time, and the time from moment (a) to moment (f) equals the total crossing time.

Figure 11 shows the time-space diagram of the bidirectional pedestrians from both sides. Before the subject and opposite pedestrians meet each other, their speeds are assumed to be the free-flow speed $v_{0}$. In order to reflect the real interaction time, the time from moment (c) to moment (e), as shown in Figure 10, is named the interaction time $\left(t_{i}\right)$ here. From moment (c) to moment (d), the resulting deceleration increases with the assumed interaction time $\left(t_{1 i}\right)$. From moment (d) to moment (e), the resulting deceleration decreases as the assumed interaction time $\left(t_{2 i}\right)$ increases. $T_{i}$ equals the sum of $t_{1 i}$ and $t_{2 i}$. At moment (e), the speeds of the subject and the opposite pedestrians return to the free-flow speed $v_{0}$.

For variable deceleration motion, the theorem of momentum and the work-energy principle are the most effective solutions. From moment (c) to moment (d), they can be expressed as in

$$
\begin{aligned}
m v_{1 i}-m v_{1} & =-\frac{1}{2} C_{\mathrm{adj}} \frac{N_{2}}{w * l_{i}} v_{0}^{2} \frac{l_{i}}{2} t_{1 i} \\
\frac{1}{2} m v_{1 i}^{2}-\frac{1}{2} m v_{1}^{2} & =-\frac{1}{2} C_{\text {adj }} \frac{N_{2}}{w * l_{i}} v_{0}^{2} \frac{l_{i}}{2} l_{i},
\end{aligned}
$$

where $m$ is the mass of the group of focal pedestrians, equal to the number of focal pedestrians, $v_{1 i}$ is the speed of the focal pedestrians at moment $(\mathrm{d})(\mathrm{m} / \mathrm{s}), t_{1 i}$ is the time from moment (c) to moment (d), and $l_{i}$ is the maximum interaction length (m).

$v_{1 i}$ and $t_{1 i}$ can be expressed as in (19) by solving (18):

$$
\begin{aligned}
& v_{1 i}^{2}=v_{0}^{2}-\frac{1}{N_{1}} C_{\text {adj }} \frac{N_{2}}{w * l_{i}} v_{0}^{2} \frac{l_{i}}{2} l_{i} \\
& t_{1 i}=\frac{2 l_{i}}{v_{1 i}+v_{0}} \\
& =\frac{2 l_{i}}{\sqrt{v_{0}^{2}-\left(1 / N_{1}\right) C_{\text {adj }}\left(N_{2} /\left(w * l_{i}\right)\right) v_{0}^{2}\left(l_{i} / 2\right) l_{i}}+v_{0}} .
\end{aligned}
$$




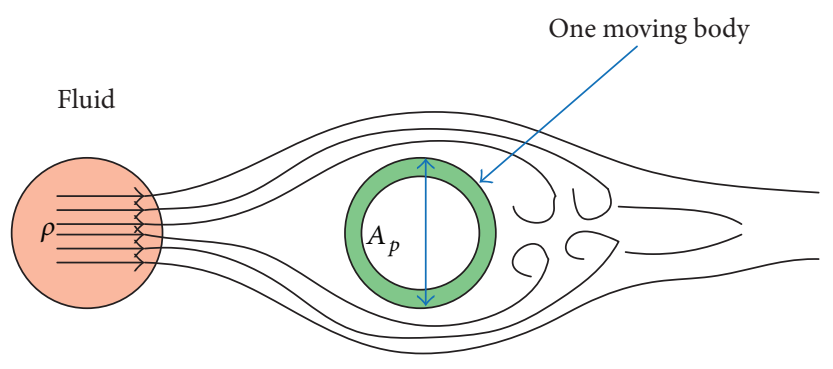

(a)

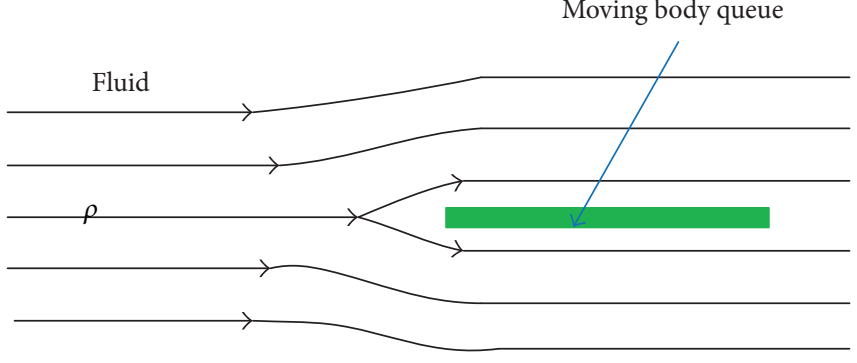

(b)

FIgURE 9: Drag force.

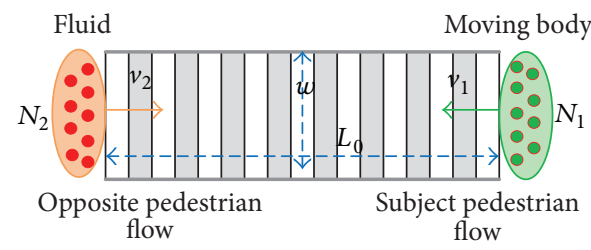

(a)

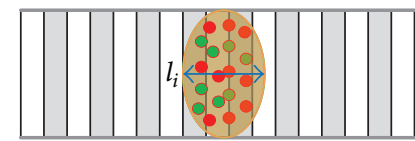

$\underset{\text { Opposite pedestrian }}{\text { flow }}$
$N_{1}+N_{2}$ Subject pedestrian
flow

(d)

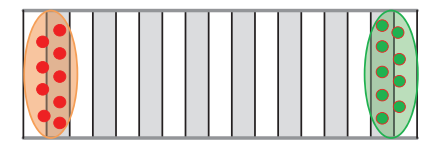

(b)

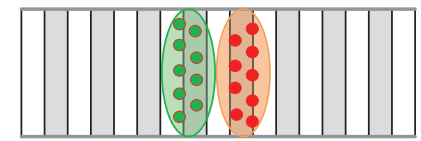

Opposite pedestrian flow

(e)

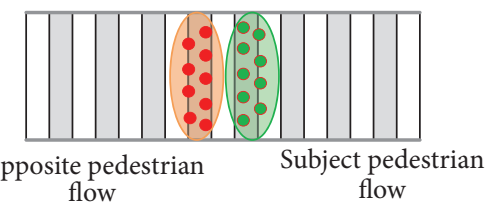

(c)

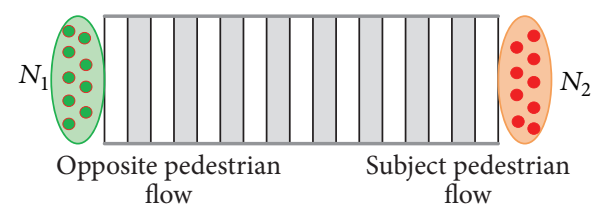

(f)

FIGURE 10: Diagram of process for a platoon of pedestrians crossing the crosswalk. $w$ : crosswalk width (m); $N_{1}$ : number of subject pedestrians; $N_{2}$ : number of opposite pedestrians; $v_{1}$ : speed of subject pedestrians $(\mathrm{m} / \mathrm{s}) ; v_{2}:$ speed of opposite pedestrians $(\mathrm{m} / \mathrm{s}) ; L_{0}:$ crosswalk length $(\mathrm{m})$; $l_{i}$ : maximum interaction length $(\mathrm{m})$.
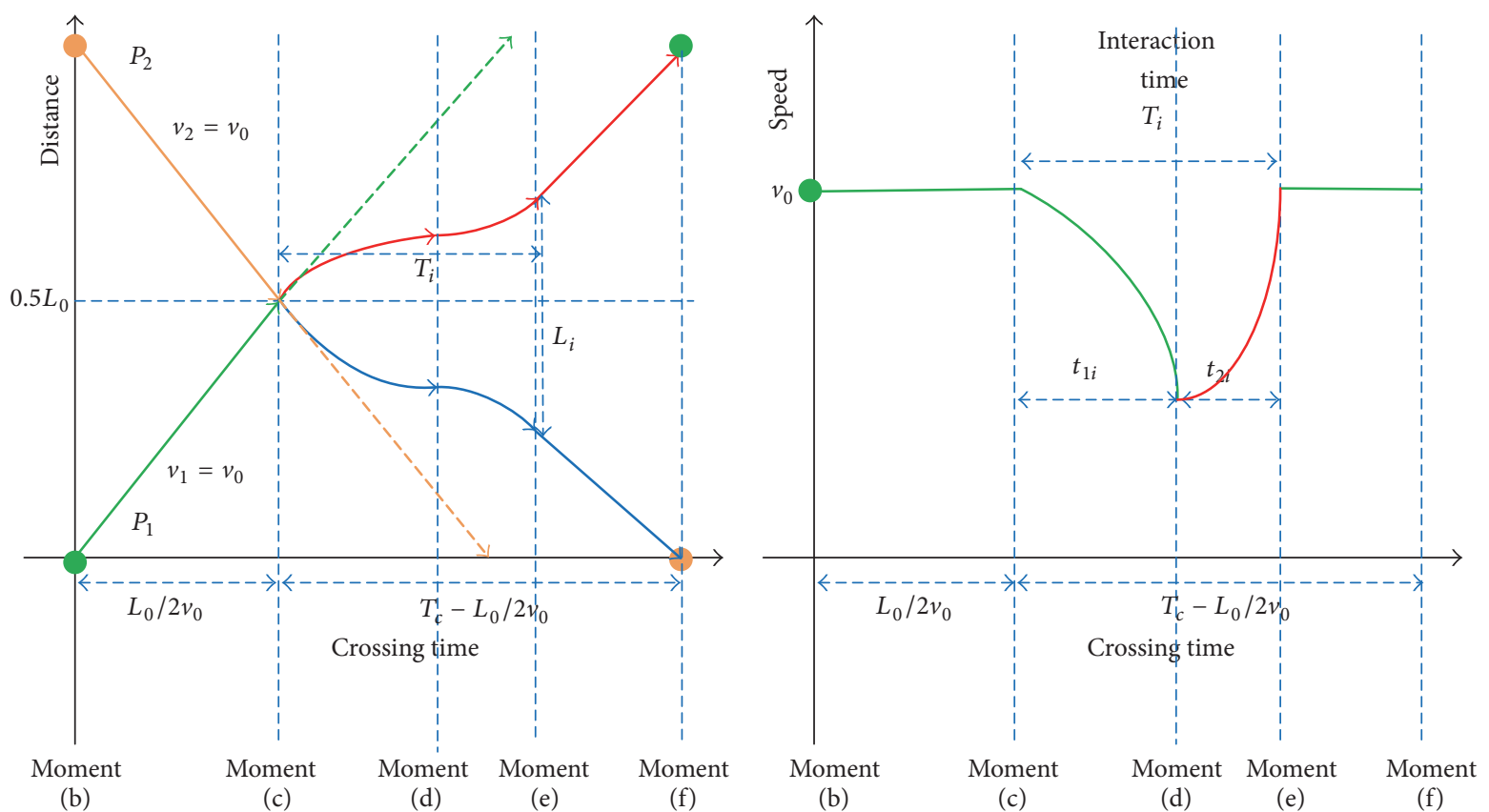

FIGURE 11: Time-space diagram for bidirectional pedestrian flows. 
Similarly, from moment (d) to moment (e), the theorem of momentum and the work-energy principle can be expressed as in

$$
\begin{aligned}
\frac{1}{2} m v_{2 i}^{2}-\frac{1}{2} m v_{1 i}^{2} & =-\frac{1}{2} C_{\text {adj }} \frac{N_{2}}{w * l_{i}} v_{1 i}^{2} \frac{l_{i}}{2} l_{i} \\
m v_{2 i}-m v_{1 i} & =-\frac{1}{2} C_{\text {adj }} \frac{N_{2}}{w * l_{i}} v_{1 i}^{2} \frac{l_{i}}{2} t_{2 i} .
\end{aligned}
$$

$v_{2 i}$ and $t_{2 i}$ can be expressed as in (21) by solving (20):

$$
v_{2 i}^{2}=v_{1 i}^{2}-\frac{1}{N_{1}} C_{\text {adj }} \frac{N_{2}}{w * l_{i}} v_{1 i}^{2} \frac{l_{i}}{2} l_{i}
$$

$$
\begin{aligned}
& t_{2 i}=\frac{2 l_{i}}{v_{1 i}+v_{2 i}} \\
& =\frac{2 l_{i}}{v_{1 i}+\sqrt{v_{1 i}^{2}-\left(1 / N_{1}\right) C_{\text {adj }}\left(N_{2} /\left(w * l_{i}\right)\right) v_{1 i}^{2}\left(l_{i} / 2\right) l_{i}}} .
\end{aligned}
$$

The drag force caused by the opposing pedestrian flow is equivalent to the resistance force that causes the deceleration. By solving the equations for the crossing time $T_{c}, T_{c}$ can be calculated as in (23):

$$
\begin{aligned}
t_{i}= & t_{1 i}+t_{2 i} \\
= & \frac{2 l_{i}}{\sqrt{v_{0}^{2}-\left(1 / N_{1}\right) C_{\text {adj }}\left(N_{2} /\left(w * l_{i}\right)\right) v_{0}^{2}\left(l_{i} / 2\right) l_{i}}+v_{0}} \\
& +\frac{2 l_{i}}{\sqrt{v_{0}^{2}-\left(1 / N_{1}\right) C_{\text {adj }}\left(N_{2} /\left(w * l_{i}\right)\right) v_{0}^{2}\left(l_{i} / 2\right) l_{i}} *\left\{1+\sqrt{1-\left(1 / N_{1}\right) C_{\text {adj }}\left(N_{2} /\left(w * l_{i}\right)\right)\left(l_{i} / 2\right) l_{i}}\right\}} \\
T_{c}= & \frac{L_{0}-2 l_{i}}{v_{0}}+t_{i} \\
= & \frac{L_{0}-2 l_{i}}{v_{0}}+\frac{2 l_{i}}{\sqrt{v_{0}^{2}-\left(1 / N_{1}\right) C_{\text {adj }}\left(N_{2} /\left(w * l_{i}\right)\right) v_{0}^{2}\left(l_{i} / 2\right) l_{i}}+v_{0}} \\
& +\frac{2 l_{i}}{\sqrt{v_{0}^{2}-\left(1 / N_{1}\right) C_{\text {adj }}\left(N_{2} /\left(w * l_{i}\right)\right) v_{0}^{2}\left(l_{i} / 2\right) l_{i}} *\left\{1+\sqrt{1-\left(1 / N_{1}\right) C_{\text {adj }}\left(N_{2} /\left(w * l_{i}\right)\right)\left(l_{i} / 2\right) l_{i}}\right\}} \\
= & \frac{L_{0}-2 l_{i}}{v_{0}}+\frac{2 l_{i}}{v_{0} \sqrt{1-C_{\text {adj }} N_{2} l_{i} / 2 N_{1} w}} .
\end{aligned}
$$

4.3. Parameters Estimation. To calculate the crossing time by applying (23), the adjusted drag coefficient $C_{\text {adj }}$ and maximum interaction length $l_{i}$ first need to be estimated according to observed data. 35 hours of video data from the seven surveyed crosswalks was analyzed. The bidirectional pedestrian flow in every cycle, the maximum interaction length, and the pedestrian crossing time in the same cycle were acquired. $v_{0}$ equals $1.45 \mathrm{~m} / \mathrm{s}$ [10]. Then, using (23), $C_{\text {adj }}$ was estimated for 187 samples. Alhajyaseen et al. [9-11] drew the conclusion that $C_{\text {adj }}$ was proportional to $r$. After analyzing the collected data, the relationship between $C_{\text {adj }}$ and $r$ was found to be as shown in Figure 12(a). $C_{\text {adj }}$ can be expressed as a function of the split ratio $r$, as shown in (24), and $r$ can be defined as in (25):

$$
\begin{aligned}
C_{\text {adj }} & =1.58 r \\
r & =\frac{N_{1}}{N_{1}+N_{2}} .
\end{aligned}
$$

Similarly, the maximum interaction length $l_{i}$ of the focal pedestrian platoon can be estimated by (26). The relationship between $l_{i}$ and $\left(N_{1}+N_{2}\right) / w$ is shown in Figure 12(b). As $\left(N_{1}+N_{2}\right) / w$ increases, $l_{i}$ also increases, and the coefficient of correlation (adjusted $R^{2}$ ) is 0.84 .

$$
l_{i}=a \frac{\left(N_{1}+N_{2}\right)}{w}=0.94 \frac{N_{1}+N_{2}}{w} .
$$

\section{Discussion and Validation}

After modeling the drag coefficient $C_{\text {adj }}$ and maximum interaction length $l_{i}$ under different crosswalk widths, (23) can be applied directly to estimate the crossing time for different bidirectional pedestrian demands. Figure 13(a) shows the relation between crossing time and crosswalk width. When the pedestrian split ratios take different fixed values, the crossing time decreases as the crosswalk width increases, and finally the crossing time approaches a constant. In other words, the pedestrian flow approaches free-flow when the crosswalk width is large enough. However, as the crosswalk width decreases, the crossing time becomes larger, because 


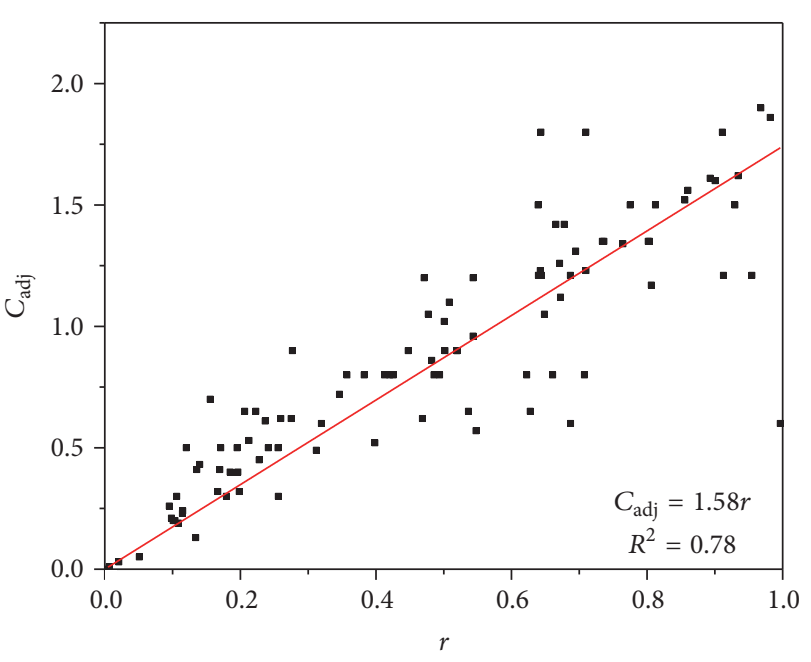

(a)

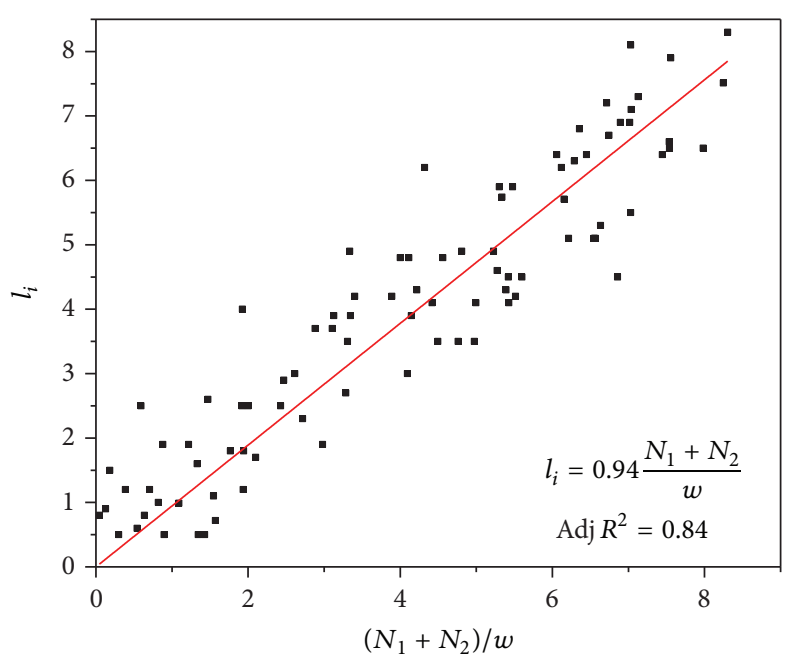

(b)

Figure 12: Modeling $C_{\text {adj }}$ and $l_{i}$.

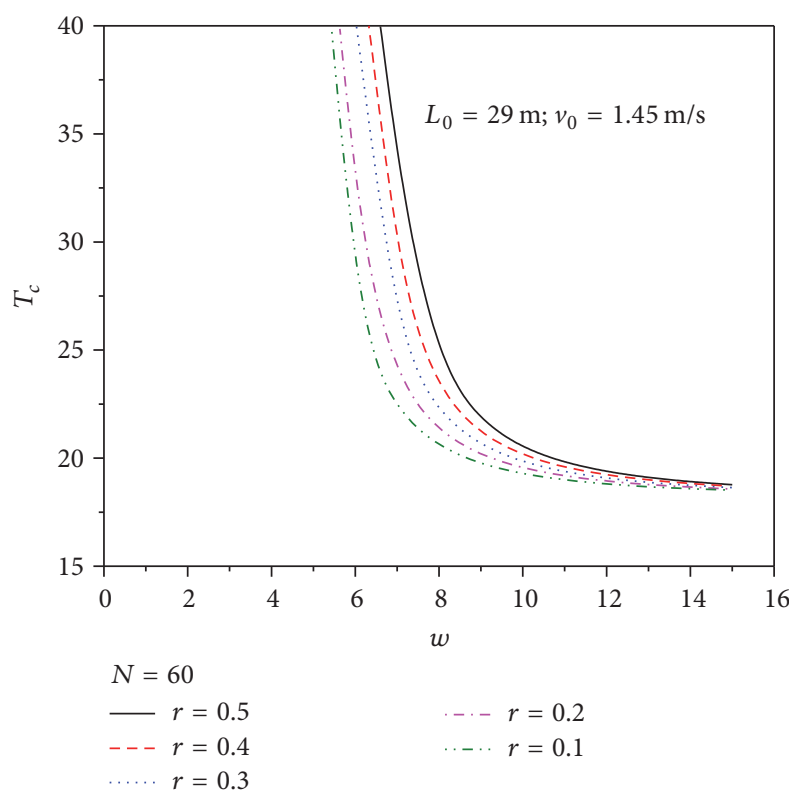

(a)

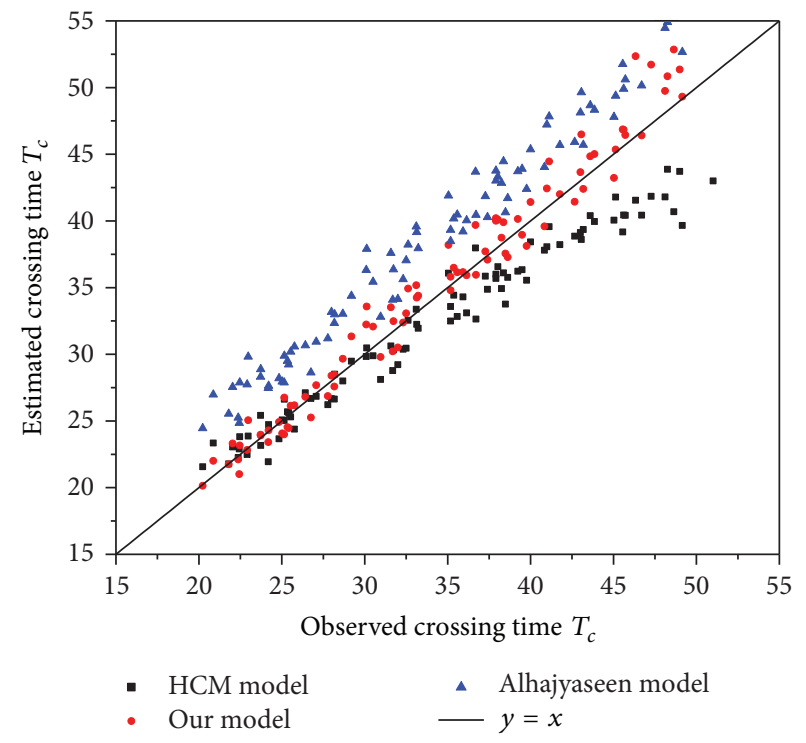

(b)

FIgURE 13: Change in $T_{c}$ with changing crosswalk width.

the interactions between the subject and opposing pedestrians become greater. When the crosswalk width is smaller than a particular value, the opposing pedestrians will definitely hinder the movement of the subject pedestrians.

To validate the established total crossing time model, the discharge times and crossing times of 187 cycles were collected at seven crosswalks. The observed total crossing times were compared with the calculated total crossing time from the established model. The differences between the observed and calculated total crossing times are shown in Figure 13(b). Based on two sample $t$-tests for the difference in waiting positions in the waiting area, at a $95 \%$ confidence level, there is no significant difference. The total crossing time
$T$ calculated using the proposed model was also compared with the total crossing time calculated using the existing model in the HCM [1], as shown in Figure 13(b). The mean absolute value of relative error of the calculated results of the proposed model was $7.38 \%$, that of the calculated results from the Alhajyaseen model was $15.26 \%$, and that of the calculated results from the HCM model was $12.42 \%$. This indicates that the proposed model is more accurate and reliable. The HCM model gives a time that is less than the observed crossing time, and the cause might be that the interactions between bidirectional pedestrians were ignored. To the contrary, the interactions between bidirectional pedestrians were overestimated, and the calculated decelerations based on the 
Alhajyaseen's model of the subject pedestrians were larger than the actual values. The calculated decelerations based on our model were also larger than the observed crossing times, but we tried to analyze the interaction process in detail and model different interaction stages. As we can see in Figure 13(b), when the observed crossing time is less than $30 \mathrm{~s}$, the total crossing time $T$ calculated by the proposed model is consistent with the empirical data and the calculated total crossing time from the existing model in the HCM. However, when the observed crossing times are more than $30 \mathrm{~s}$, the proposed model is more consistent with the observed data. In other words, the proposed model is more effective at high-occupancy crosswalks.

\section{Conclusion}

This paper introduces a modified method to model the discharge time and crossing time considering bidirectional pedestrian flow at signalized crosswalks. Traffic wave and drag force theories are used to model the discharge time and the crossing time, respectively. The discharge time takes the characteristics of the waiting position distribution into consideration, and the proposed model satisfies the need for the longest queue to discharge. Based on the drag force theory, the model of the crossing time $T_{c}$ is a function of the crosswalk width, the number of bidirectional pedestrians, and maximum interaction length and speed. The maximum interaction length $l_{i}$ is also modeled based on empirical data. But the effects of the traffic signal control of pedestrian on the pedestrian speed were ignored. Therefore, a continuation of this study will quantify that factor. The key step in modeling an accurate crossing time is to estimate parameters accurately. Thus, it is necessary that a large amount of data be gathered and analyzed in order that the accuracy of the drag coefficient can be improved. The proposed models provide guidance for the design of crosswalk width and pedestrian green intervals.

\section{Conflicts of Interest}

The authors declare that they have no conflicts of interest.

\section{References}

[1] Highway Capacity Manual 2010, Transportation Research Board of the National Academies, Washington, DC, USA, 2010.

[2] A. Golani and H. Damti, "Model for estimating crossing times at high-occupancy crosswalks," Transportation Research Record: Journal of the Transportation Research Board, vol. 2002, pp. 125130, 2007.

[3] W. H. K. Lam, J. Y. S. Lee, K. S. Chan, and P. K. Goh, "A generalised function for modeling bi-directional flow effects on indoor walkways in Hong Kong," Transportation Research Part A: Policy and Practice, vol. 37, no. 9, pp. 789-810, 2003.

[4] W. K. M. Alhajyaseen, H. Nakamura, and M. Asano, "Effects of bi-directional pedestrian flow characteristics upon the capacity of signalized crosswalks," in Proceedings of the 6th International Symposium on Highway Capacity and Quality of Service (ISHC '11), pp. 526-535, July 2011.
[5] S. Marisamynathan and P. Vedagiri, "Modeling pedestrian delay at signalized intersection crosswalks under mixed traffic condition," Procedia-Social and Behavioral Sciences, vol. 104, pp. 708-717, 2013.

[6] X. Zhang, P. Chen, and H. Nakamura, "Modeling pedestrian walking speed at signalized crosswalks considering crosswalk length and signal timing," in Proceedings of the 10th International Conference of the Eastern Asia for Transportation Studies (EASTS '13), pp. 1-15, Committee of EASTS, Taipei, Taiwan, 2013.

[7] L.-J. Xu and W. Wang, "Model of pedestrians crossing time at signalized intersection," Journal of Traffic and Transportation Engineering, vol. 5, no. 1, pp. 111-115, 2005.

[8] H.-T. Zhao and D. Guo, "Model of pedestrians crossing time at signalized intersection with exclusive pedestrian phase," Journal of Harbin Institute of Technology, vol. 44, no. 8, pp. 57-61, 2012.

[9] W. Alhajyaseen, H. Nakamura, and J. Catbagan, "A proposed methodology for modeling pedestrian crossing time at signalized crosswalks considering Bi-directional flow," Proceedings of Infrastructure Planning, vol. 38, 2008.

[10] W. Alhajyaseen and H. Nakamura, "A methodology for modeling pedestrian platoon discharge and crossing times at signalized crosswalks," in Proceedings of the 88th Annual Meeting of the Transportation Research Board, Compendium of Papers CDROM, pp. 11-15, Washington, DC, USA, 2009.

[11] W. Alhajyaseen and H. Nakamura, "Estimating the minimum required width of signalized crosswalks considering bidirectional pedestrian flow and different age groups," Journal of the Eastern Asia Society for Transportation Studies (EASTS), vol. 1, no. 2, pp. 181-198, 2010.

[12] C. Walck, "Hand-book on statistical distributions for experimentalists," Internal Report SUF-PFY/96-01, University of Stockholm, Stockholm, Sweden, 2007.

[13] L. G. C. E. Pugh, "The influence of wind resistance in running and walking and the mechanical efficiency of work against horizontal or vertical forces," The Journal of Physiology, vol. 213, no. 2, pp. 255-276, 1971.

[14] L. G. C. E. Pugh, “The relation of oxygen intake and speed in competition cycling and comparative observations on the bicycle ergometer," The Journal of Physiology, vol. 241, no. 3, pp. 795-808, 1974. 


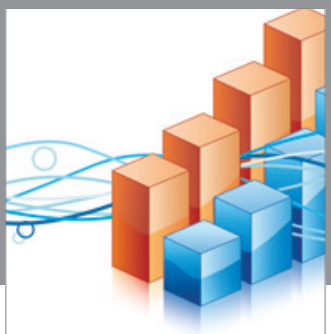

Advances in

Operations Research

vatem alat4

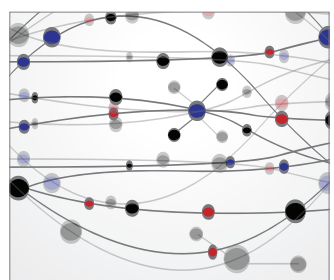

\section{The Scientific} World Journal
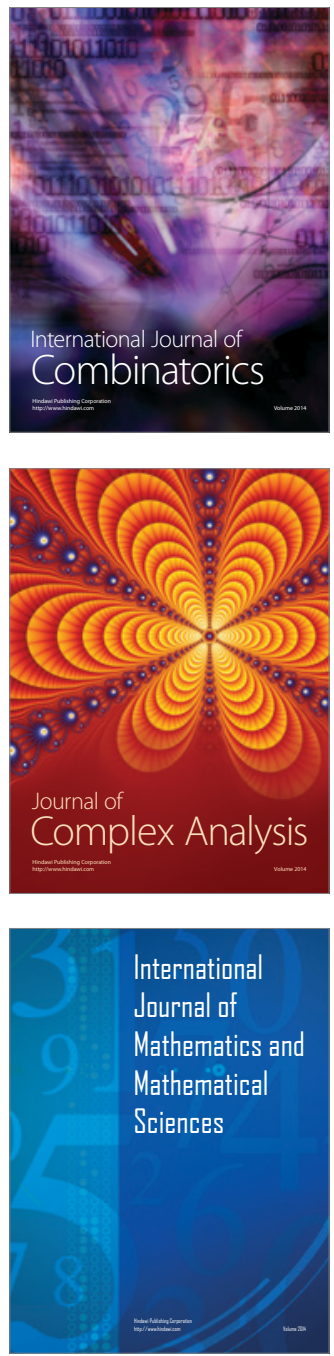
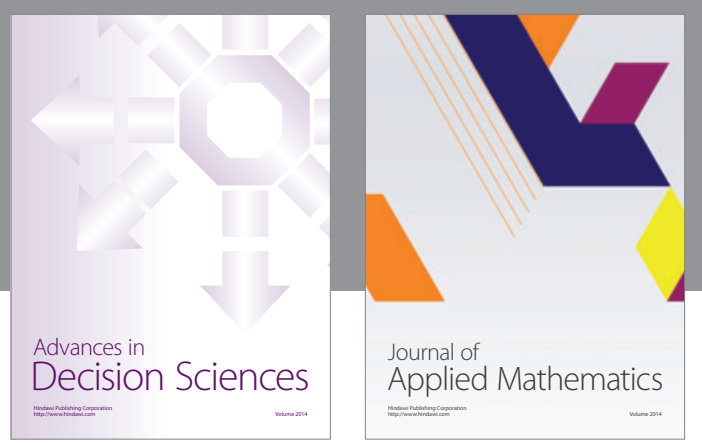

Algebra

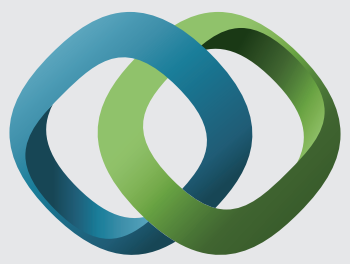

\section{Hindawi}

Submit your manuscripts at

https://www.hindawi.com
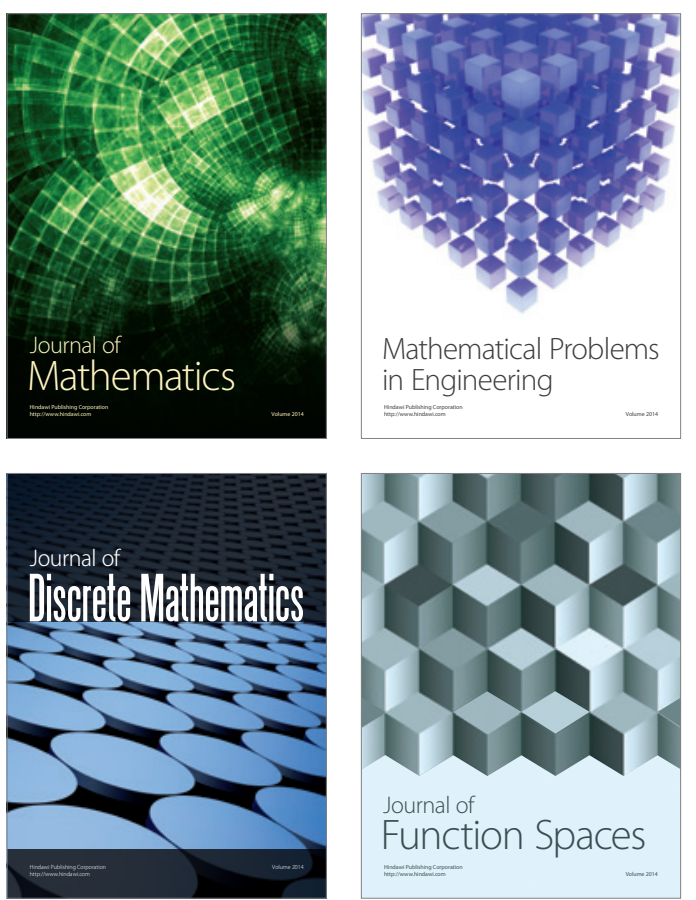

Mathematical Problems in Engineering
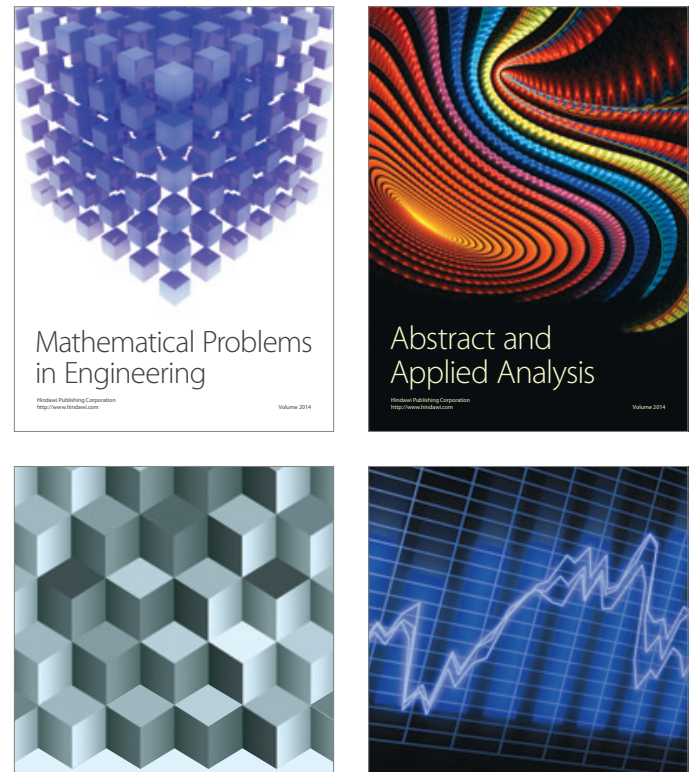

Journal of

Function Spaces

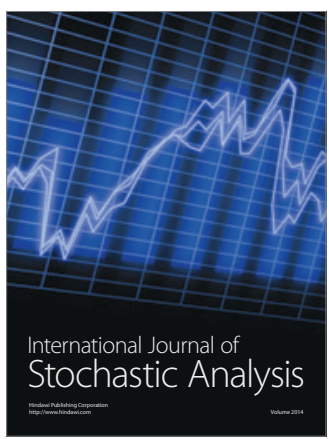

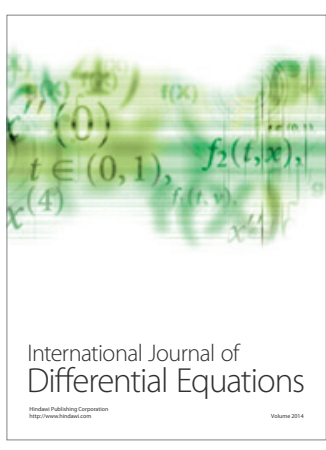
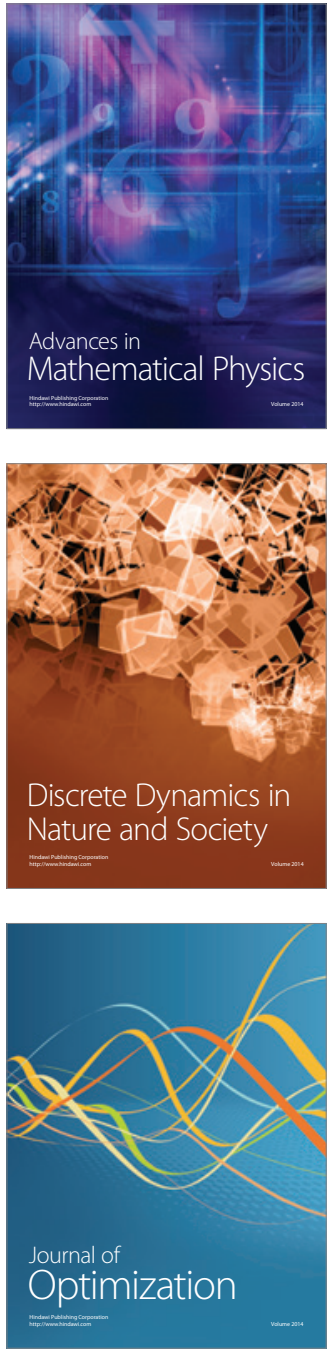